\title{
MAJORIZATION TYPE INEQUALITIES VIA GREEN FUNCTION AND HERMITE'S POLYNOMIAL
}

\author{
M. Adil Khan ${ }^{1}$, N. LAtif ${ }^{2}$ And J. PeČARIĆ ${ }^{3}$ \\ ${ }^{1}$ Department of Mathematics, University of Peshawar, \\ Peshawar 25000, Pakistan \\ adilswati@gmail.com \\ ${ }^{2}$ Department of Mathematics, Govt. College University, \\ Faisalabad 38000, Pakistan \\ naveed707@gmail.com \\ ${ }^{3}$ Faculty of Textile Technology, University of Zagreb, \\ Prilaz baruna Filipovića 28a, 10000 Zagreb, Croatia \\ pecaric@element.hr
}

\begin{abstract}
The Hermite polynomial and Green function are used to construct the identities related to majorization type inequalities for convex function. By using $\check{C}$ ebyšev functional the bounds for the new identities are found to develop the Grüss and Ostrowski type inequalities. Further more exponential convexity together with Cauchy means is presented for linear functionals associated with the obtained inequalities.

Key words and Phrases: Majorization theorem, Hermite's interpolating polynomial, $(m, n-m)$ interpolating polynomial, two-point Taylor interpolating polynomial, $\check{C}$ ebyšev functional, $n$-exponentially convex function, mean value theorems, Stolarsky type means.
\end{abstract}

\begin{abstract}
Abstrak. Polinom Hermite dan Fungsi Green digunakan untuk mengkonstruksi identitas yang berkaitan dengan membuat majorisasi jenis pertidaksamaan untuk Fungsi Konveks. Dengan menggunakan Fungsional $\breve{C}$ ebyšev, batas untuk identitas baru yang ditemukan digunakan untuk dibangun jenis pertidaksamaan Grüss dan Ostrowski. Selanjutnya Konveksitas Eksponensial dengan rata-rata Cauchy disajikan untuk fungsional-fungsional linear yang berasosiasi dengan pertidaksamaan yang diperoleh.

Kata kunci: Majorization theorem, Hermite's interpolating polynomial, ( $m, n-$ $m$ ) interpolating polynomial, two-point Taylor interpolating polynomial, Čebyšev functional, $n$-exponentially convex function, mean value theorems, Stolarsky type means.
\end{abstract}

2000 Mathematics Subject Classification: 26D15, 26D20, 26D99.

Received: 10-07-2015, revised: 19-11-2015, accepted: 19-11-2015. 


\section{INTRODUCTION}

For fixed $m \geq 2$ let

$$
\mathbf{x}=\left(x_{1}, \ldots, x_{m}\right), \mathbf{y}=\left(y_{1}, \ldots, y_{m}\right)
$$

denote two real $m$-tuples. Let

$$
\begin{gathered}
x_{[1]} \geq x_{[2]} \geq \ldots \geq x_{[m]}, \quad y_{[1]} \geq y_{[2]} \geq \ldots \geq y_{[m]}, \\
x_{(1)} \leq x_{(2)} \leq \ldots \leq x_{(m)}, \quad y_{(1)} \leq y_{(2)} \leq \ldots \leq y_{(m)}
\end{gathered}
$$

be their ordered components.

Definition 1.1. [21, p. 319] $\boldsymbol{x}$ is said to majorize $\boldsymbol{y}$ (or $\boldsymbol{y}$ is said to be majorized by $\boldsymbol{x})$, in symbol, $\boldsymbol{x} \succ \boldsymbol{y}$, if

$$
\sum_{i=1}^{l} y_{[i]} \leq \sum_{i=1}^{l} x_{[i]}
$$

holds for $l=1,2, \ldots, m-1$ and

$$
\sum_{i=1}^{m} x_{i}=\sum_{i=1}^{m} y_{i}
$$

Note that (1) is equivalent to

$$
\sum_{i=m-l+1}^{m} y_{(i)} \leq \sum_{i=m-l+1}^{m} x_{(i)}
$$

holds for $l=1,2, \ldots, m-1$.

The following theorem is well-known as the majorization theorem given by Marshall and Olkin [19, p. 14] (see also [21, p. 320]):

Theorem 1.2. Let $\boldsymbol{x}=\left(x_{1}, \ldots, x_{m}\right), \boldsymbol{y}=\left(y_{1}, \ldots, y_{m}\right)$ be two m-tuples such that $x_{i}$, $y_{i} \in[\alpha, \beta](i=1, \ldots, m)$. Then

$$
\sum_{i=1}^{m} \phi\left(y_{i}\right) \leq \sum_{i=1}^{m} \phi\left(x_{i}\right)
$$

holds for every continuous convex function $\phi:[\alpha, \beta] \rightarrow \mathbb{R}$ if and only if $\mathbf{x} \succ \mathbf{y}$ holds.

The following theorem can be regarded as a weighted version of Theorem 1.2 and is proved by Fuchs in [14] ([19, p. 580], [21, p. 323]):

Theorem 1.3. Let $\boldsymbol{x}=\left(x_{1}, \ldots, x_{m}\right), \boldsymbol{y}=\left(y_{1}, \ldots, y_{m}\right)$ be two decreasing real $m$-tuples with $x_{i}, y_{i} \in[\alpha, \beta](i=1, \ldots, m)$ and $\mathbf{w}=\left(w_{1}, w_{2}, \ldots, w_{m}\right)$ be a real $m$-tuple such that

$$
\sum_{i=1}^{l} w_{i} y_{i} \leq \sum_{i=1}^{l} w_{i} x_{i} \text { for } l=1, \ldots, m-1
$$


and

$$
\sum_{i=1}^{m} w_{i} y_{i}=\sum_{i=1}^{m} w_{i} x_{i}
$$

Then for every continuous convex function $\phi:[\alpha, \beta] \rightarrow \mathbb{R}$, we have

$$
\sum_{i=1}^{m} w_{i} \phi\left(y_{i}\right) \leq \sum_{i=1}^{m} w_{i} \phi\left(x_{i}\right)
$$

The following integral version of Theorem 1.3 is a simple consequence of Theorem 12.14 in [23] (see also [21, p.328]):

Theorem 1.4. Let $x, y:[a, b] \rightarrow[\alpha, \beta]$ be decreasing and $w:[a, b] \rightarrow \mathbb{R}$ be continuous functions. If

$$
\int_{a}^{\nu} w(t) y(t) d t \leq \int_{a}^{\nu} w(t) x(t) d t \text { for every } \nu \in[a, b],
$$

and

$$
\int_{a}^{b} w(t) y(t) d t=\int_{a}^{b} w(t) x(t) d t
$$

hold, then for every continuous convex function $\phi:[\alpha, \beta] \rightarrow \mathbb{R}$, we have

$$
\int_{a}^{b} w(t) \phi(y(t)) d t \leq \int_{a}^{b} w(t) \phi(x(t)) d t
$$

For other integral version and generalization of majorization theorem see [19, p. 583], [1]-[8], [10, 17, 18, 20, 23].

Consider the Green function $G$ defined on $[\alpha, \beta] \times[\alpha, \beta]$ by

$$
G(t, s)= \begin{cases}\frac{(t-\beta)(s-\alpha)}{\beta-\alpha}, & \alpha \leq s \leq t ; \\ \frac{(s-\beta)(t-\alpha)}{\beta-\alpha}, & t \leq s \leq \beta .\end{cases}
$$

The function $G$ is convex in $s$, it is symmetric, so it is also convex in $t$. The function $G$ is continuous in $s$ and continuous in $t$.

For any function $\phi:[\alpha, \beta] \rightarrow \mathbb{R}, \phi \in C^{2}([\alpha, \beta])$, we can easily show by integrating by parts that the following is valid

$$
\phi(x)=\frac{\beta-x}{\beta-\alpha} \phi(\alpha)+\frac{x-\alpha}{\beta-\alpha} \phi(\beta)+\int_{\alpha}^{\beta} G(x, s) \phi^{\prime \prime}(s) d s,
$$

where the function $G$ is defined as above in (9) ([26]).

Let $-\infty<\alpha<\beta<\infty$ and $\alpha \leq a_{1}<a_{2} \cdots<a_{r} \leq \beta,(r \geq 2)$ be the given points. For $\phi \in C^{n}[\alpha, \beta]$ a unique polynomial $\rho_{H}(s)$ of degree $(n-1)$ exists satisfying any of the following conditions:

Hermite conditions:

$$
\rho_{H}^{(i)}\left(a_{j}\right)=\phi^{(i)}\left(a_{j}\right) ; 0 \leq i \leq k_{j}, 1 \leq j \leq r, \sum_{j=1}^{r} k_{j}+r=n .
$$


It is of great interest to note that Hermite conditions include the following particular cases:

Type $(m, n-m)$ conditions: $\left(r=2,1 \leq m \leq n-1, k_{1}=m-1, k_{2}=n-m-1\right)$

$$
\begin{gathered}
\rho_{(m, n)}^{(i)}(\alpha)=\phi^{(i)}(\alpha), 0 \leq i \leq m-1, \\
\rho_{(m, n)}^{(i)}(\beta)=\phi^{(i)}(\beta), 0 \leq i \leq n-m-1,
\end{gathered}
$$

Two-point Taylor conditions: $\left(n=2 m, r=2, k_{1}=k_{2}=m-1\right)$

$$
\rho_{2 T}^{(i)}(\alpha)=\phi^{(i)}(\alpha), \rho_{2 T}^{(i)}(\beta)=\phi^{(i)}(\beta), 0 \leq i \leq m-1 .
$$

We have the following result from [9].

Theorem 1.5. Let $-\infty<\alpha<\beta<\infty$ and $\alpha \leq a_{1}<a_{2} \cdots<a_{r} \leq \beta$, $(r \geq 2)$ be the given points, and $\phi \in C^{n}([\alpha, \beta])$. Then we have

$$
\phi(t)=\rho_{H}(t)+R_{H, n}(\phi, t)
$$

where $\rho_{H}(t)$ is the Hermite interpolating polynomial, i.e.

$$
\rho_{H}(t)=\sum_{j=1}^{r} \sum_{i=0}^{k_{j}} H_{i j}(t) \phi^{(i)}\left(a_{j}\right) ;
$$

the $H_{i j}$ are fundamental polynomials of the Hermite basis defined by

$$
\begin{gathered}
H_{i j}(t)=\left.\frac{1}{i !} \frac{\omega(t)}{\left(t-a_{j}\right)^{k_{j}+1-i}} \sum_{k=0}^{k_{j}-i} \frac{1}{k !} \frac{d^{k}}{d t^{k}}\left(\frac{\left(t-a_{j}\right)^{k_{j}+1}}{\omega(t)}\right)\right|_{t=a_{j}}\left(t-a_{j}\right)^{k}, \\
\omega(t)=\prod_{j=1}^{r}\left(t-a_{j}\right)^{k_{j}+1},
\end{gathered}
$$

and the remainder is given by

$$
R_{H, n}(\phi, t)=\int_{\alpha}^{\beta} G_{H, n}(t, s) \phi^{(n)}(s) d s
$$

where $G_{H, n}(t, s)$ is defined by

$$
G_{H, n}(t, s)=\left\{\begin{array}{l}
\sum_{j=1}^{l} \sum_{i=0}^{k_{j}} \frac{\left(a_{j}-s\right)^{n-i-1}}{(n-i-1) !} H_{i j}(t) ; s \leq t, \\
-\sum_{j=l+1}^{r} \sum_{i=0}^{k_{j}} \frac{\left(a_{j}-s\right)^{n-i-1}}{(n-i-1) !} H_{i j}(t) ; s \geq t,
\end{array}\right.
$$

for all $a_{l} \leq s \leq a_{l+1} ; l=0, \ldots, r$ with $a_{0}=\alpha$ and $a_{r+1}=\beta$.

Remark 1.1. In particular cases,

for type $(m, n-m)$ conditions, from Theorem 1.5 we have

$$
\phi(t)=\rho_{(m, n)}(t)+R_{(m, n)}(\phi, t)
$$


where $\rho_{(m, n)}(t)$ is $(m, n-m)$ interpolating polynomial, i.e

with

$$
\rho_{(m, n)}(t)=\sum_{i=0}^{m-1} \tau_{i}(t) \phi^{i}(\alpha)+\sum_{i=0}^{n-m-1} \eta_{i}(t) \phi^{i}(\beta),
$$

$$
\tau_{i}(t)=\frac{1}{i !}(t-\alpha)^{i}\left(\frac{t-\beta}{\alpha-\beta}\right)^{n-m} \sum_{k=0}^{m-1-i}\left(\begin{array}{c}
n-m+k-1 \\
k
\end{array}\right)\left(\frac{t-\alpha}{\beta-\alpha}\right)^{k}
$$

and

$$
\eta_{i}(t)=\frac{1}{i !}(t-\beta)^{i}\left(\frac{t-\alpha}{\beta-\alpha}\right)^{m} \sum_{k=0}^{n-m-1-i}\left(\begin{array}{c}
m+k-1 \\
k
\end{array}\right)\left(\frac{t-\beta}{\alpha-\beta}\right)^{k} .
$$

and the remainder $R_{(m, n)}(\phi, t)$ is given by

$$
R_{(m, n)}(\phi, t)=\int_{\alpha}^{\beta} G_{(m, n)}(t, s) \phi^{(n)}(s) d s
$$

with

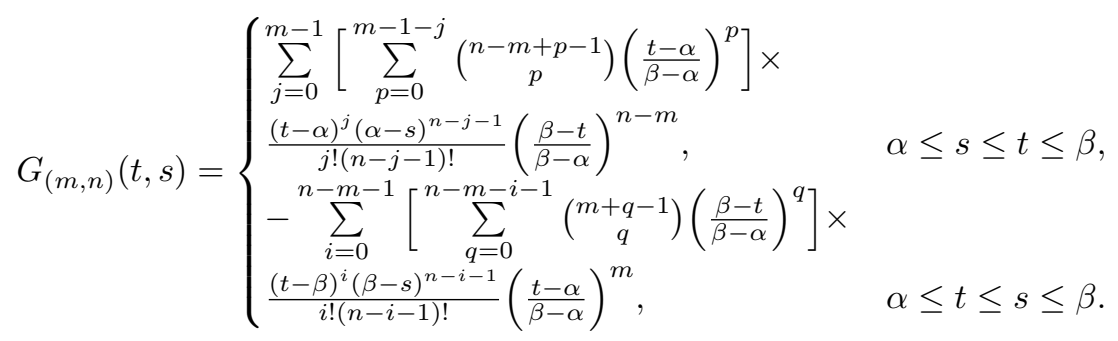

For Type Two-point Taylor conditions, from Theorem 1.5 we have

$$
\phi(t)=\rho_{2 T}(t)+R_{2 T}(\phi, t)
$$

where $\rho_{2 T}(t)$ is the two-point Taylor interpolating polynomial i.e,

$$
\begin{array}{r}
\rho_{2 T}(t)=\sum_{i=0}^{m-1} \sum_{k=0}^{m-1-i}\left(\begin{array}{c}
m+k-1 \\
k
\end{array}\right)\left[\frac{(t-\alpha)^{i}}{i !}\left(\frac{t-\beta}{\alpha-\beta}\right)^{m}\left(\frac{t-\alpha}{\beta-\alpha}\right)^{k} \phi^{(i)}(\alpha)\right. \\
\left.+\frac{(t-\beta)^{i}}{i !}\left(\frac{t-\alpha}{\beta-\alpha}\right)^{m}\left(\frac{t-\beta}{\alpha-\beta}\right)^{k} \phi^{(i)}(\beta)\right]
\end{array}
$$

and the remainder $R_{2 T}(\phi, t)$ is given by

$$
R_{2 T}(\phi, t)=\int_{\alpha}^{\beta} G_{2 T}(t, s) \phi^{(n)}(s) d s
$$

with

$$
G_{2 T}(t, s)= \begin{cases}\frac{(-1)^{m}}{(2 m-1) !} p^{m}(t, s) \sum_{j=0}^{m-1}\left(\begin{array}{c}
m-1+j \\
j
\end{array}\right)(t-s)^{m-1-j} q^{j}(t, s), & s \leq t \\
\frac{(-1)^{m}}{(2 m-1) !} q^{m}(t, s) \sum_{j=0}^{m-1}\left(\begin{array}{c}
m-1+j \\
j
\end{array}\right)(s-t)^{m-1-j} p^{j}(t, s), & s \geq t\end{cases}
$$


where $p(t, s)=\frac{(s-\alpha)(\beta-t)}{\beta-\alpha}, q(t, s)=p(s, t), \forall t, s \in[\alpha, \beta]$.

The following Lemma describes the positivity of Green's function (14) see (Beesack [11] and [Levin [24]).

Lemma 1.6. The Green's function $G_{H, n}(t, s)$ has the following properties:

(i) $\frac{G_{H, n}(t, s)}{w(t)}>0, a_{1} \leq t \leq a_{r}, a_{1}<s<a_{r}$;

(ii) $G_{H, n}(t, s) \leq \frac{1}{(n-1) !(\beta-\alpha)}|w(t)|$;

(iii) $\int_{\alpha}^{\beta} G_{H, n}(t, s) d s=\frac{w(t)}{n !}$.

In order to recall the definition of $n$-convex function, first we write the definition of divided difference.

Definition 1.7. [21, p. 15] Let $\phi$ be a real-valued function defined on $[\alpha, \beta]$. The divided difference of order $n$ of the function $\phi$ at distinct points $[\alpha, \beta]$ is defined recursively by

$$
\phi\left[x_{i}\right]=\phi\left(x_{i}\right), \quad(i=0, \ldots, n)
$$

and

$$
\phi\left[x_{0}, \ldots, x_{n}\right]=\frac{\phi\left[x_{1}, \ldots, x_{n}\right]-\phi\left[x_{0}, \ldots, x_{n-1}\right]}{x_{n}-x_{0}} .
$$

The value $\phi\left[x_{0}, \ldots, x_{n}\right]$ is independent of the order of the points $x_{0}, \ldots, x_{n}$.

The definition may be extended to include the case that some (or all) the points coincide. Assuming that $\phi^{(j-1)}(x)$ exists, we define

$$
\phi \underbrace{[x, \ldots, x]}_{j-\text { times }}=\frac{\phi^{(j-1)}(x)}{(j-1) !} .
$$

Definition 1.8. $[21$, p. 15] A function $\phi:[\alpha, \beta] \rightarrow \mathbb{R}$ is said to be $n$-convex, $n \geq 0$, on $[\alpha, \beta]$ if and only if for all choices of $(n+1)$ distinct points $x_{0}, \ldots, x_{n} \in[\alpha, \beta]$, the nth order divided difference is non negative that is

$$
\phi\left[x_{0}, x_{1}, \ldots, x_{n}\right] \geq 0 .
$$

Theorem 1.9. $\left[21\right.$, p. 16] Let $\phi:[\alpha, \beta] \rightarrow \mathbb{R}$ be a function such that $\phi^{(n)}$ exists, then $\phi$ is $n$-convex if and only if $\phi^{(n)} \geq 0$.

We arrange the paper in this manner, in section 2, we use Hermite interpolating polynomial and Green function to establish identities for majorization inequalities. We present generalized majorization inequalities and in particular we discuss the results for $(m, n-m)$ interpolating polynomial, two-point Taylor interpolating polynomial. In section 3, we give bounds for the identities related to the generalizations of majorization inequalities by using $\check{C}$ ebyšev functionals. We also 
give Grüss type inequalities and Ostrowski-type inequalities for these functionals. In section 4, we present Lagrange and Cauchy type mean value theorems related to the defined functionals and also give $n$-exponential convexity which leads to exponential convexity and then log-convexity. At the end, in section 5, we discuss some families of functions which enable us to construct a large families of functions that are exponentially convex and also give Stolarsky type means with their monotonicity.

\section{Generalization of Majorization Inequalities}

We begin this section with the proof of some identities related to generalizations of majorization inequality.

Theorem 2.1. Let $-\infty<\alpha<\beta<\infty$ and $\alpha \leq a_{1}<a_{2} \cdots<a_{r} \leq \beta,(r \geq 2)$ be the given points, and $\phi \in C^{n}([\alpha, \beta])$ and $\boldsymbol{w}=\left(w_{1}, \ldots, w_{m}\right), \boldsymbol{x}=\left(x_{1}, \ldots, x_{m}\right)$ and $\boldsymbol{y}=\left(y_{1}, \ldots, y_{m}\right)$ be $m$-tuples such that $x_{l}, y_{l} \in[\alpha, \beta], w_{l} \in \mathbb{R}(l=1, \ldots, m)$. Also let $H_{i j}, G_{H, n}$ and $G$ be as defined in (12), (14) and (9) respectively. Then

$$
\begin{aligned}
& \sum_{l=1}^{m} w_{l} \phi\left(x_{l}\right)-\sum_{l=1}^{m} w_{l} \phi\left(y_{l}\right)=\frac{\phi(\beta)-\phi(\alpha)}{\beta-\alpha} \sum_{l=1}^{m} w_{l}\left(x_{l}-y_{l}\right) \\
& +\int_{\alpha}^{\beta}\left[\sum_{l=1}^{m} w_{l}\left(G\left(x_{l}, t\right)-G\left(y_{l}, t\right)\right)\right] \sum_{j=1}^{r} \sum_{i=0}^{k_{j}} \phi^{(i+2)}\left(a_{j}\right) H_{i j}(t) d t \\
& +\int_{\alpha}^{\beta} \int_{\alpha}^{\beta}\left[\sum_{l=1}^{m} w_{l}\left(G\left(x_{l}, t\right)-G\left(y_{l}, t\right)\right)\right] G_{H, n-2}(t, s) \phi^{(n)}(s) d s d t .
\end{aligned}
$$

Proof. Use (10) in $\sum_{l=1}^{m} w_{l} \phi\left(x_{l}\right)-\sum_{l=1}^{m} w_{l} \phi\left(y_{l}\right)$ we have

$$
\begin{aligned}
\sum_{l=1}^{m} w_{l} \phi\left(x_{l}\right)-\sum_{l=1}^{m} w_{l} \phi\left(y_{l}\right) & \\
& =\frac{\phi(\beta)-\phi(\alpha)}{\beta-\alpha} \sum_{l=1}^{m} w_{l}\left(x_{l}-y_{l}\right)+\int_{\alpha}^{\beta}\left[\sum_{l=1}^{m} w_{l} G\left(x_{l}, t\right)-\sum_{l=1}^{m} w_{l} G\left(y_{l}, t\right)\right] \phi^{\prime \prime}(t) d t .
\end{aligned}
$$

By Theorem 1.5, $\phi^{\prime \prime}(t)$ can be expressed as

$$
\phi^{\prime \prime}(t)=\sum_{j=1}^{r} \sum_{i=0}^{k_{j}} H_{i j}(t) \phi^{(i+2)}\left(a_{j}\right)+\int_{\alpha}^{\beta} G_{H, n-2}(t, s) \phi^{(n)}(s) d s .
$$

Using (25) in (24) we get (23). 
Integral version of the above theorem can be stated as:

Theorem 2.2. Let $-\infty<\alpha<\beta<\infty$ and $\alpha \leq a_{1}<a_{2} \cdots<a_{r} \leq \beta,(r \geq 2)$ be the given points, $\phi \in C^{n}([\alpha, \beta])$ and $x, y:[a, b] \rightarrow[\alpha, \beta], w:[a, b] \rightarrow \mathbb{R}$ be continuous functions. Also let $H_{i j}, G_{H, n}$ and $G$ be as defined in (12), (14) and (9) respectively. Then

$$
\begin{aligned}
& \int_{a}^{b} w(\tau) \phi(x(\tau)) d \tau-\int_{a}^{b} w(\tau) \phi(y(\tau)) d \tau=\frac{\phi(\beta)-\phi(\alpha)}{\beta-\alpha} \int_{a}^{b} w(\tau)(x(\tau)-y(\tau)) d \tau \\
& +\int_{\alpha}^{\beta}\left[\int_{a}^{b} w(\tau)(G(x(\tau), t)-G(y(\tau), t)) d \tau\right] \sum_{j=1}^{r} \sum_{i=0}^{k_{j}} \phi^{(i+2)}\left(a_{j}\right) H_{i j}(t) d t \\
& +\int_{\alpha}^{\beta} \int_{\alpha}^{\beta}\left[\int_{a}^{b} w(\tau)(G(x(\tau), t)-G(y(\tau), t)) d \tau\right] G_{H, n-2}(t, s) \phi^{(n)}(s) d s d t .
\end{aligned}
$$

Theorem 2.3. Let $-\infty<\alpha=a_{1}<a_{2} \cdots<a_{r}=\beta<\infty,(r \geq 2)$ be the given points, $\boldsymbol{w}=\left(w_{1}, \ldots, w_{m}\right), \boldsymbol{x}=\left(x_{1}, \ldots, x_{m}\right)$ and $\boldsymbol{y}=\left(y_{1}, \ldots, y_{m}\right)$ be $m$-tuples such that $x_{l}, y_{l} \in[\alpha, \beta], w_{l} \in \mathbb{R}(l=1, \ldots, m)$ and $H_{i j}, G$ be as defined in (12) and (9) respectively. Let $\phi:[\alpha, \beta] \rightarrow \mathbb{R}$ be $n$-convex and

$$
\sum_{l=1}^{m} w_{l}\left(G\left(x_{l}, t\right)-G\left(y_{l}, t\right)\right) \geq 0, \quad t \in[\alpha, \beta] .
$$

Consider the inequality

$$
\begin{aligned}
& \sum_{l=1}^{m} w_{l} \phi\left(x_{l}\right)-\sum_{l=1}^{m} w_{l} \phi\left(y_{l}\right) \geq \frac{\phi(\beta)-\phi(\alpha)}{\beta-\alpha} \sum_{l=1}^{m} w_{l}\left(x_{l}-y_{l}\right) \\
& +\int_{\alpha}^{\beta}\left[\sum_{l=1}^{m} w_{l}\left(G\left(x_{l}, t\right)-G\left(y_{l}, t\right)\right)\right] \sum_{j=1}^{r} \sum_{i=0}^{k_{j}} \phi^{(i+2)}\left(a_{j}\right) H_{i j}(t) d t .
\end{aligned}
$$

(i) If $k_{j}$ is odd for each $j=2, . ., r$, then the inequality (28) holds.

(ii) If $k_{j}$ is odd for each $j=2, . ., r-1$ and $k_{r}$ is even, then the reverse inequality in (28) holds.

Proof. (i) Since the function $\phi$ is $n$-convex, therefore without loss of generality we can assume that $\phi$ is $n$-times differentiable and $\phi^{(n)} \geq 0$ see $[21$, p. 16 and p. 293]. Also as it is given that $k_{j}$ is odd for each $j=2, \ldots, r$, therefore we have $\omega(t) \geq 0$ and by using Lemma $1.6(\mathrm{i})$ we have $G_{H, n-2}(t, s) \geq 0$. Hence, we can apply Theorem 2.1 to obtain (28).

(ii) If $k_{r}$ is even then $\left(t-a_{r}\right)^{k_{r}+1} \leq 0$ for any $t \in[\alpha, \beta]$. Also clearly $(t-$ $\left.a_{1}\right)^{k_{1}+1} \geq 0$ for any $t \in[\alpha, \beta]$ and $\prod_{j=2}^{r-1}\left(t-a_{j}\right)^{k_{j}+1} \geq 0$ for $t \in[\alpha, \beta]$ if $k_{j}$ is odd for each $j=2, . ., r-1$, therefore combining all these we have 
$\omega(t)=\prod_{j=1}^{r}\left(t-a_{j}\right)^{k_{j}+1} \leq 0$ for any $t \in[\alpha, \beta]$ and by using Lemma 1.6(i) we have $G_{H, n-2}(t, s) \leq 0$. Hence, we can apply Theorem 2.1 to obtain reverse inequality in (28).

Integral version of the above theorem can be stated as:

Theorem 2.4. Let $-\infty<\alpha=a_{1}<a_{2} \cdots<a_{r}=\beta<\infty,(r \geq 2)$ be given points and $x, y:[a, b] \rightarrow[\alpha, \beta], w:[a, b] \rightarrow \mathbb{R}$ be continuous functions and $H_{i j}$ and $G$ be as defined in (12) and (9) respectively. Let $\phi:[\alpha, \beta] \rightarrow \mathbb{R}$ be $n$-convex and

$$
\int_{a}^{b} w(\tau)(G(x(\tau), t)-G(y(\tau), t)) d \tau \geq 0, \quad t \in[\alpha, \beta] .
$$

Consider the inequality

$$
\begin{aligned}
& \int_{a}^{b} w(\tau) \phi(x(\tau)) d \tau-\int_{a}^{b} w(\tau) \phi(y(\tau)) d \tau \geq \frac{\phi(\beta)-\phi(\alpha)}{\beta-\alpha} \int_{a}^{b} w(\tau)(x(\tau)-y(\tau)) d \tau \\
& +\int_{\alpha}^{\beta}\left[\int_{a}^{b} w(\tau)(G(x(\tau), t)-G(y(\tau), t)) d \tau\right] \sum_{j=1}^{r} \sum_{i=0}^{k_{j}} \phi^{(i+2)}\left(a_{j}\right) H_{i j}(t) d t .
\end{aligned}
$$

(i) If $k_{j}$ is odd for each $j=2, . ., r$, then the inequality (30) holds.

(ii) If $k_{j}$ is odd for each $j=2, . ., r-1$ and $k_{r}$ is even, then the reverse inequality in (30) holds.

By using type $(m, n-m)$ conditions we can give the following result.

Corollary 2.5. Let $[\alpha, \beta]$ be an interval and $\boldsymbol{w}=\left(w_{1}, \ldots, w_{p}\right), \boldsymbol{x}=\left(x_{1}, \ldots, x_{p}\right)$ and $\boldsymbol{y}=\left(y_{1}, \ldots, y_{p}\right)$ be $p$-tuples such that $x_{l}, y_{l} \in[\alpha, \beta], w_{l} \in \mathbb{R}(l=1, \ldots, p)$. Let $G$ be the green function as defined in (9) and $\tau_{i}, \eta_{i}$ be as defined in (16) and (17) respectively. Let $\phi:[\alpha, \beta] \rightarrow \mathbb{R}$ be $n$-convex and the inequality (27) holds for p-tuples. Consider the inequality

$$
\begin{aligned}
\sum_{l=1}^{p} w_{l} \phi & \left(x_{l}\right)-\sum_{l=1}^{p} w_{l} \phi\left(y_{l}\right) \\
\geq & \frac{\phi(\beta)-\phi(\alpha)}{\beta-\alpha} \sum_{l=1}^{p} w_{l}\left(x_{l}-y_{l}\right) \\
+ & \int_{\alpha}^{\beta}\left[\sum_{l=1}^{p} w_{l}\left(G\left(x_{l}, t\right)-G\left(y_{l}, t\right)\right)\right] \\
& \left(\sum_{i=0}^{m-1} \tau_{i}(t) \phi^{(i+2)}(\alpha)+\sum_{i=0}^{n-m-1} \eta_{i}(t) \phi^{(i+2)}(\beta)\right) d t .
\end{aligned}
$$

(i) If $n-m$ is even, then the inequality (31) holds.

(ii) If $n-m$ is odd, then the reverse inequality in (31) holds. 
By using Two-point Taylor conditions we can give the following result.

Corollary 2.6. Let $[\alpha, \beta]$ be an interval, $\boldsymbol{w}=\left(w_{1}, \ldots, w_{p}\right), \boldsymbol{x}=\left(x_{1}, \ldots, x_{p}\right)$ and $\boldsymbol{y}=\left(y_{1}, \ldots, y_{p}\right)$ be $p$-tuples such that $x_{l}, y_{l} \in[\alpha, \beta], w_{l} \in \mathbb{R}(l=1, \ldots, p)$ and $G$ be the green function as defined in (9). Let $\phi:[\alpha, \beta] \rightarrow \mathbb{R}$ be $n$-convex and the inequality (27) holds for p-tuples. Consider the inequality

$$
\begin{aligned}
& \sum_{l=1}^{p} w_{l} \phi\left(x_{l}\right)-\sum_{l=1}^{p} w_{l} \phi\left(y_{l}\right) \\
& \geq \frac{\phi(\beta)-\phi(\alpha)}{\beta-\alpha} \sum_{l=1}^{p} w_{l}\left(x_{l}-y_{l}\right)+\int_{\alpha}^{\beta}\left[\sum_{l=1}^{p} w_{l}\left(G\left(x_{l}, t\right)-G\left(y_{l}, t\right)\right)\right] \\
& {\left[\sum _ { i = 0 } ^ { m - 1 } \sum _ { k = 0 } ^ { m - 1 - i } ( \begin{array} { c } 
{ m + k - 1 } \\
{ k }
\end{array} ) \left[\frac{(t-\alpha)^{i}}{i !}\left(\frac{t-\beta}{\alpha-\beta}\right)^{m}\left(\frac{t-\alpha}{\beta-\alpha}\right)^{k} \phi^{(i+2)}(\alpha)\right.\right.} \\
&\left.\left.+\frac{(t-\beta)^{i}}{i !}\left(\frac{t-\alpha}{\beta-\alpha}\right)^{m}\left(\frac{t-\beta}{\alpha-\beta}\right)^{k} \phi^{(i+2)}(\beta)\right]\right] d t .
\end{aligned}
$$

(i) If $m$ is even, then the inequality (32) holds.

(ii) If $m$ is odd, then the reverse inequality in (32) holds.

Remark 2.1. Similarly we can give integral version of Corollaries 2.5,2.6.

The following generalization of majorization theorem is valid.

Theorem 2.7. Let $-\infty<\alpha=a_{1}<a_{2} \cdots<a_{r}=\beta<\infty,(r \geq 2)$ be the given points, $\boldsymbol{x}=\left(x_{1}, \ldots, x_{m}\right)$ and $\boldsymbol{y}=\left(y_{1}, \ldots, y_{m}\right)$ be $m$-tuples such that $\mathbf{y} \prec \mathbf{x}$ with $x_{l}$, $y_{l} \in[\alpha, \beta](l=1, \ldots, m)$. Let $H_{i j}$ be as defined in (12) and $\phi:[\alpha, \beta] \rightarrow \mathbb{R}$ be $n$-convex. Consider

$$
\begin{aligned}
& \sum_{l=1}^{m} \phi\left(x_{l}\right)- \sum_{l=1}^{m} \phi\left(y_{l}\right) \\
& \geq \int_{\alpha}^{\beta}\left[\sum_{l=1}^{m}\left(G\left(x_{l}, t\right)-G\left(y_{l}, t\right)\right)\right] \\
& \\
& \sum_{j=1}^{r} \sum_{i=0}^{k_{j}} \phi^{(i+2)}\left(a_{j}\right) H_{i j}(t) d t .
\end{aligned}
$$

(i) If $k_{j}$ is odd for each $j=2, . ., r$, then the inequality (33) holds.

(ii) If $k_{j}$ is odd for each $j=2, . ., r-1$ and $k_{r}$ is even, then the reverse inequality in (33) holds.

If the inequality (reverse inequality) in (33) holds and the function $F()=$. $\sum_{j=1}^{r} \sum_{i=0}^{k_{j}} \phi^{(i+2)}\left(a_{j}\right) H_{i j}($.$) is non negative ( non positive), then the right hand side of$ 
(33) will be non negative (non positive) that is the inequality (reverse inequality) in (2) will holds.

Proof. (i) Since the function $G$ is convex and $\mathbf{y} \prec \mathbf{x}$ therefore by Theorem 1.2, the inequality (27) holds for $w_{l}=1$. Hence by Theorem 2.3(i) the inequality (33) holds. Also if the function $F$ is convex then by using $F$ in (2) instead of $\phi$ we get that the right hand side of (33) is non negative.

Similarly we can prove part (ii).

In the following theorem we give generalization of Fuch's majorization theorem.

Theorem 2.8. Let $-\infty<\alpha=a_{1}<a_{2} \cdots<a_{r}=\beta<\infty,(r \geq 2)$ be the given points, $\boldsymbol{x}=\left(x_{1}, \ldots, x_{m}\right)$ and $\boldsymbol{y}=\left(y_{1}, \ldots, y_{m}\right)$ be decreasing $m$-tuples and $\boldsymbol{w}=$ $\left(w_{1}, \ldots, w_{m}\right)$ be any $m$-tuple with $x_{l}, y_{l} \in[\alpha, \beta], w_{l} \in \mathbb{R}(l=1, \ldots, m)$ which satisfy (3) and (4). Let $H_{i j}$ be as defined in (12) and $\phi:[\alpha, \beta] \rightarrow \mathbb{R}$ be $n$-convex, then

$$
\begin{aligned}
& \sum_{l=1}^{m} w_{l} \phi\left(x_{l}\right)-\sum_{l=1}^{m} w_{l} \phi\left(y_{l}\right) \\
& \quad \geq \int_{\alpha}^{\beta}\left[\sum_{l=1}^{m} w_{l}\left(G\left(x_{l}, t\right)-G\left(y_{l}, t\right)\right)\right] \sum_{j=1}^{r} \sum_{i=0}^{k_{j}} \phi^{(i+2)}\left(a_{j}\right) H_{i j}(t) d t .
\end{aligned}
$$

(i) If $k_{j}$ is odd for each $j=2, . ., r$, then the inequality (34) holds.

(ii) If $k_{j}$ is odd for each $j=2, . ., r-1$ and $k_{r}$ is even, then the reverse inequality in (34) holds.

If the inequality (reverse inequality) in (34) holds and the function $F()=$. $\sum_{j=1}^{r} \sum_{i=0}^{k_{j}} \phi^{(i+2)}\left(a_{j}\right) H_{i j}($.$) is non negative (non postive), then the right hand side of$ (34) will be non negative (non positive) that is the inequality (reverse inequality) in (5) will hold.

Proof. Similar to the proof of Theorem 2.7 .

In the following theorem we give generalized majorization integral inequality.

Theorem 2.9. Let $-\infty<\alpha=a_{1}<a_{2} \cdots<a_{r}=\beta<\infty,(r \geq 2)$ be the given points, and $x, y:[a, b] \rightarrow[\alpha, \beta]$ be decreasing and $w:[a, b] \rightarrow \mathbb{R}$ be continuous functions such that (6) and (7) hold. Also let $H_{i j}$ be as defined in (12) and $\phi$ : 
$[\alpha, \beta] \rightarrow \mathbb{R}$ be $n$-convex and consider the inequlity

$$
\begin{aligned}
& \int_{a}^{b} w(\tau) \phi(x(\tau)) d \tau-\int_{a}^{b} w(\tau) \phi(y(\tau)) d \tau \\
& \geq \int_{\alpha}^{\beta}\left[\int_{a}^{b} w(\tau)(G(x(\tau), t)-G(y(\tau), t)) d \tau\right] \sum_{j=1}^{r} \sum_{i=0}^{k_{j}} \phi^{(i+2)}\left(a_{j}\right) H_{i j}(t) d t .
\end{aligned}
$$

(i) If $k_{j}$ is odd for each $j=2, . ., r$, then the inequality (35) holds.

(ii) If $k_{j}$ is odd for each $j=2, . ., r-1$ and $k_{r}$ is even, then the reverse inequality in (35) holds.

If the inequality (reverse inequality) in (35) holds and the function

$$
F(.)=\sum_{j=1}^{r} \sum_{i=0}^{k_{j}} \phi^{(i+2)}\left(a_{j}\right) H_{i j}(.)
$$

is non negative (non positive), then the right hand side of (35) will be non negative (non positive) that is the inequality (reverse inequality) in (8) will hold.

By using type $(m, n-m)$ conditions we can give generalization of majorization inequality for majorized tuples:

Corollary 2.10. Let $[\alpha, \beta]$ be an interval, $\boldsymbol{x}=\left(x_{1}, \ldots, x_{p}\right)$ and $\boldsymbol{y}=\left(y_{1}, \ldots, y_{p}\right)$ be any $p$-tuple such that $\mathbf{y} \prec \mathbf{x}$ with $x_{l}, y_{l} \in[\alpha, \beta](l=1, \ldots, p)$. Let $\tau_{i}$ and $\eta_{i}$ be as defined in (16) and (17) respectively and $\phi:[\alpha, \beta] \rightarrow \mathbb{R}$ be $n$-convex. Consider

$$
\begin{aligned}
\sum_{l=1}^{p} \phi & \left(x_{l}\right)-\sum_{l=1}^{p} \phi\left(y_{l}\right) \\
& \geq \int_{\alpha}^{\beta}\left[\sum_{l=1}^{p}\left(G\left(x_{l}, t\right)-G\left(y_{l}, t\right)\right)\right]\left(\sum_{i=0}^{m-1} \tau_{i}(t) \phi^{(i+2)}(\alpha)+\sum_{i=0}^{n-m-1} \eta_{i}(t) \phi^{(i+2)}(\beta)\right) d t .
\end{aligned}
$$

(i) If $n-m$ is even, then the inequality (36) holds.

(ii) If $n-m$ is odd, then the reverse inequality in (36) holds.

If the inequality (reverse inequality) in (36) holds and the function

$$
F(.)=\sum_{i=0}^{m-1} \phi^{(i+2)}(\alpha) \tau_{i}(.)+\sum_{i=0}^{n-m-1} \phi^{(i+2)}(\beta) \eta_{i}(.)
$$

is non negative (non positive), then the right hand side of (36) will be non negative (non positive) that is the inequality (reverse inequality) in (2) will hold.

By using Two-point Taylor conditions we can give generalization of majorization inequality for majorized tuples: 
Corollary 2.11. Let $[\alpha, \beta]$ be an interval and $\boldsymbol{x}=\left(x_{1}, \ldots, x_{p}\right), \boldsymbol{y}=\left(y_{1}, \ldots, y_{p}\right)$ be decreasing $p$-tuples such that $\mathbf{y} \prec \mathbf{x}$ with $x_{l}, y_{l} \in[\alpha, \beta](l=1, \ldots, p)$. Let $\phi:[\alpha, \beta] \rightarrow$ $\mathbb{R}$ be $n$-convex. Consider

$$
\sum_{l=1}^{p} \phi\left(x_{l}\right)-\sum_{l=1}^{p} \phi\left(y_{l}\right) \geq \int_{\alpha}^{\beta}\left[\sum_{l=1}^{p}\left(G\left(x_{l}, t\right)-G\left(y_{l}, t\right)\right)\right] F(t) d t
$$

$$
\text { where } \begin{aligned}
F(t)=\sum_{i=0}^{m-1} \sum_{k=0}^{m-1-i}\left(\begin{array}{c}
m+k-1 \\
k
\end{array}\right) & {\left[\frac{(t-\alpha)^{i}}{i !}\left(\frac{t-\beta}{\alpha-\beta}\right)^{m}\left(\frac{t-\alpha}{\beta-\alpha}\right)^{k} \phi^{(i+2)}(\alpha)\right.} \\
+ & \left.\frac{(t-\beta)^{i}}{i !}\left(\frac{t-\alpha}{\beta-\alpha}\right)^{m}\left(\frac{t-\beta}{\alpha-\beta}\right)^{k} \phi^{(i+2)}(\beta)\right] .
\end{aligned}
$$

(i) If $m$ is even, then the inequality (37) holds.

(ii) If $m$ is odd, then the reverse inequality in (37) holds.

If the inequality (reverse inequality) in (37) holds and the function $F($.$) is non$ negative (non positive), then the right hand side of (37) will be non negative (non positive) that is the inequality (reverse inequality) in (2) will hold.

By using type $(m, n-m)$ conditions we can give the following weighted majorization inequality.

Corollary 2.12. Let $[\alpha, \beta]$ be an interval and $\boldsymbol{x}=\left(x_{1}, \ldots, x_{p}\right)$ and $\boldsymbol{y}=\left(y_{1}, \ldots, y_{p}\right)$ be decreasing $p$-tuples and $\boldsymbol{w}=\left(w_{1}, \ldots, w_{p}\right)$ be any $p$-tuple such that $x_{l}, y_{l} \in[\alpha, \beta], w_{l} \in$ $\mathbb{R}(l=1, \ldots, p)$ which satisfy (3) and (4). Let $\tau_{i}$ and $\eta_{i}$ be as defined in (16) and (17) respectively and let $\phi:[\alpha, \beta] \rightarrow \mathbb{R}$ be $n$-convex. Consider the inequality

$$
\begin{aligned}
& \sum_{l=1}^{p} w_{l} \phi\left(x_{l}\right)-\sum_{l=1}^{p} w_{l} \phi\left(y_{l}\right) \\
& \geq \int_{\alpha}^{\beta}\left[\sum_{l=1}^{p} w_{l}\left(G\left(x_{l}, t\right)-G\left(y_{l}, t\right)\right)\right] \\
&\left(\sum_{i=0}^{m-1} \tau_{i}(t) \phi^{(i+2)}(\alpha)+\sum_{i=0}^{n-m-1} \eta_{i}(t) \phi^{(i+2)}(\beta)\right) d t .
\end{aligned}
$$

(i) If $n-m$ is even, then the inequality (38) holds.

(ii) If $n-m$ is odd, then the reverse inequality in (38) holds.

If the inequality (reverse inequality) in (38) holds and the function

$$
F(.)=\sum_{i=0}^{m-1} \phi^{(i+2)}(\alpha) \tau_{i}(.)+\sum_{i=0}^{n-m-1} \phi^{(i+2)}(\beta) \eta_{i}(.)
$$

is non negative (non positive), then the right hand side of (38) will be non negative (non positive) that is the inequality (reverse inequality) in (5) will hold. 
By using Two-point Taylor conditions we can give the following weighted majorization inequality.

Corollary 2.13. Let $[\alpha, \beta]$ be an interval and $\boldsymbol{x}=\left(x_{1}, \ldots, x_{p}\right), \boldsymbol{y}=\left(y_{1}, \ldots, y_{p}\right)$ be decreasing $p$-tuples such that $x_{l}, y_{l} \in[\alpha, \beta], w_{l} \in \mathbb{R}(l=1, \ldots, p)$ which satisfy (3) and (4) and let $\phi:[\alpha, \beta] \rightarrow \mathbb{R}$ be $n$-convex. Consider the inequality

$$
\begin{aligned}
\sum_{l=1}^{p} w_{l} \phi\left(x_{l}\right)-\sum_{l=1}^{p} w_{l} \phi\left(y_{l}\right) \geq \int_{\alpha}^{\beta} & {\left[\sum_{l=1}^{p} w_{l}\left(G\left(x_{l}, t\right)-G\left(y_{l}, t\right)\right)\right] F(t) d t, } \\
\text { where } F(t)=\sum_{i=0}^{m-1} \sum_{k=0}^{m-1-i}\left(\begin{array}{c}
m+k-1 \\
k
\end{array}\right) & {\left[\frac{(t-\alpha)^{i}}{i !}\left(\frac{t-\beta}{\alpha-\beta}\right)^{m}\left(\frac{t-\alpha}{\beta-\alpha}\right)^{k} \phi^{(i+2)}(\alpha)\right.} \\
+ & \left.\frac{(t-\beta)^{i}}{i !}\left(\frac{t-\alpha}{\beta-\alpha}\right)^{m}\left(\frac{t-\beta}{\alpha-\beta}\right)^{k} \phi^{(i+2)}(\beta)\right] .
\end{aligned}
$$

(i) If $m$ is even, then the inequality (39) holds.

(ii) If $m$ is odd, then the reverse inequality in (39) holds.

If the inequality (reverse inequality) in (39) holds and the function $F($.$) is non$ negative (non positive), then the right hand side of (39) will be non negative (non positive) that is the inequality (reverse inequality) in (5) will hold.

The integral version of the above Corollaries can be stated as:

Corollary 2.14. Let $[\alpha, \beta]$ be an interval and $x, y:[a, b] \rightarrow[\alpha, \beta]$ be decreasing and $w:[a, b] \rightarrow \mathbb{R}$ be continuous function such that (6), (7) hold. Let $\tau_{i}$ and $\eta_{i}$ be as defined in (16) and (17) respectively and $\phi:[\alpha, \beta] \rightarrow \mathbb{R}$ be $n$-convex. Consider

$$
\begin{aligned}
& \int_{a}^{b} w(\tau) \phi(x(\tau)) d \tau-\int_{a}^{b} w(\tau) \phi(y(\tau)) d \tau \\
& \geq \int_{\alpha}^{\beta}\left[\int_{a}^{b} w(\tau)(G(x(\tau), t)-G(y(\tau), t)) d \tau\right] \\
&\left(\sum_{i=0}^{m-1} \tau_{i}(t) \phi^{(i+2)}(\alpha)+\sum_{i=0}^{n-m-1} \eta_{i}(t) \phi^{(i+2)}(\beta)\right) d t .
\end{aligned}
$$

(i) If $n-m$ is even, then the inequality (40) holds.

(ii) If $n-m$ is odd, then the reverse inequality in (40) holds.

If the inequality (reverse inequality) in (40) holds and the function

$$
F(.)=\sum_{i=0}^{m-1} \phi^{(i+2)}(\alpha) \tau_{i}(.)+\sum_{i=0}^{n-m-1} \phi^{(i+2)}(\beta) \eta_{i}(.)
$$

is non negative (non positive), then the right hand side of (40) will be non negative (non positive) that is the inequality (reverse inequality) in (8) will hold. 
Corollary 2.15. Let $[\alpha, \beta]$ be an interval and $x, y:[a, b] \rightarrow[\alpha, \beta]$ be decreasing and $w:[a, b] \rightarrow \mathbb{R}$ be continuous functions such that (6) and (7) hold. Let $\phi:[\alpha, \beta] \rightarrow \mathbb{R}$ be $n$-convex. Consider the inequality

$$
\begin{gathered}
\int_{a}^{b} w(\tau) \phi(x(\tau)) d \tau-\int_{a}^{b} w(\tau) \phi(y(\tau)) d \tau \\
\geq \int_{\alpha}^{\beta}\left[\int_{a}^{b} w(\tau)(G(x(\tau), t)-G(y(\tau), t)) d \tau\right] F(t) d t \\
\text { where } F(t)=\sum_{i=0}^{m-1} \sum_{k=0}^{m-1-i}\left(\begin{array}{c}
m+k-1 \\
k
\end{array}\right)\left[\frac{(t-\alpha)^{i}}{i !}\left(\frac{t-\beta}{\alpha-\beta}\right)^{m}\left(\frac{t-\alpha}{\beta-\alpha}\right)^{k} \phi^{(i+2)}(\alpha)\right. \\
\left.+\frac{(t-\beta)^{i}}{i !}\left(\frac{t-\alpha}{\beta-\alpha}\right)^{m}\left(\frac{t-\beta}{\alpha-\beta}\right)^{k} \phi^{(i+2)}(\beta)\right] .
\end{gathered}
$$

(i) If $m$ is even, then the inequality (41) holds.

(ii) If $m$ is odd, then the reverse inequality in (41) holds.

If the inequality (reverse inequality) in (41) holds and the function $F($.$) is non$ negative (non positive), then the right hand side of (41) will be non negative (non positive) that is the inequality (reverse inequality) in (8) will hold.

\section{Bounds for Identities Related to Generalizations of Majorization} INEQUALITY

For two Lebesgue integrable functions $f, h:[\alpha, \beta] \rightarrow \mathbb{R}$ we consider the Čebyšev functional

$$
\Lambda(f, h)=\frac{1}{\beta-\alpha} \int_{\alpha}^{\beta} f(t) h(t) d t-\frac{1}{\beta-\alpha} \int_{\alpha}^{\beta} f(t) d t \cdot \frac{1}{\beta-\alpha} \int_{\alpha}^{\beta} h(t) d t .
$$

In [13] the authors proved the following theorems:

Theorem 3.1. Let $f:[\alpha, \beta] \rightarrow \mathbb{R}$ be a Lebesgue integrable function and $h:[\alpha, \beta] \rightarrow$ $\mathbb{R}$ be an absolutely continuous function with $(\cdot-\alpha)(\beta-\cdot)\left[h^{\prime}\right]^{2} \in L[\alpha, \beta]$. Then we have the inequality

$$
|\Lambda(f, h)| \leq \frac{1}{\sqrt{2}}[\Lambda(f, f)]^{\frac{1}{2}} \frac{1}{\sqrt{\beta-\alpha}}\left(\int_{\alpha}^{\beta}(x-\alpha)(\beta-x)\left[h^{\prime}(x)\right]^{2} d x\right)^{\frac{1}{2}} .
$$

The constant $\frac{1}{\sqrt{2}}$ in (42) is the best possible. 
Theorem 3.2. Assume that $h:[\alpha, \beta] \rightarrow \mathbb{R}$ is monotonic nondecreasing on $[\alpha, \beta]$ and $f:[\alpha, \beta] \rightarrow \mathbb{R}$ is absolutely continuous with $f^{\prime} \in L_{\infty}[\alpha, \beta]$. Then we have the inequality

$$
|\Lambda(f, h)| \leq \frac{1}{2(\beta-\alpha)}\left\|f^{\prime}\right\|_{\infty} \int_{\alpha}^{\beta}(x-\alpha)(\beta-x) d h(x) .
$$

The constant $\frac{1}{2}$ in (43) is the best possible.

In the sequel we use the above theorems to obtain generalizations of the results proved in the previous section.

For $m$-tuples $\mathbf{w}=\left(w_{1}, \ldots, w_{m}\right), \mathbf{x}=\left(x_{1}, \ldots, x_{m}\right)$ and $\mathbf{y}=\left(y_{1}, \ldots, y_{m}\right)$ with $x_{l}$, $y_{l} \in[\alpha, \beta], w_{l} \in \mathbb{R}(l=1, \ldots, m)$ and the Green functions $G$ and $G_{H, n}$ be as defined in (9) and (14) respectively, denote

$$
\mathfrak{L}(s)=\int_{\alpha}^{\beta}\left[\sum_{l=1}^{m} w_{l}\left(G\left(x_{l}, t\right)-G\left(y_{l}, t\right)\right)\right] G_{H, n-2}(t, s) d t, \quad s \in[\alpha, \beta],
$$

similarly for continuous functions $x, y:[a, b] \rightarrow[\alpha, \beta], w:[a, b] \rightarrow \mathbb{R}$ and the Green function $G$ and $G_{H, n}$ be as defined in (9) and (14) respectively, denote

$$
\mathfrak{J}(s)=\int_{\alpha}^{\beta}\left[\int_{a}^{b} w(\tau)(G(x(\tau), t)-G(y(\tau), t)) d \tau\right] G_{H, n-2}(t, s) d t, \quad s \in[\alpha, \beta] .
$$

Consider the Čebyšev functionals $\Lambda(\mathfrak{L}, \mathfrak{L}), \Lambda(\mathfrak{J}, \mathfrak{J})$ are given by:

$$
\begin{aligned}
& \Lambda(\mathfrak{L}, \mathfrak{L})=\frac{1}{\beta-\alpha} \int_{\alpha}^{\beta} \mathfrak{L}^{2}(s) d s-\left(\frac{1}{\beta-\alpha} \int_{\alpha}^{\beta} \mathfrak{L}(s) d s\right)^{2}, \\
& \Lambda(\mathfrak{J}, \mathfrak{J})=\frac{1}{\beta-\alpha} \int_{\alpha}^{\beta} \mathfrak{J}^{2}(s) d s-\left(\frac{1}{\beta-\alpha} \int_{\alpha}^{\beta} \mathfrak{J}(s) d s\right)^{2} .
\end{aligned}
$$

Theorem 3.3. Let $-\infty<\alpha<\beta<\infty$ and $\alpha \leq a_{1}<a_{2} \cdots<a_{r} \leq \beta,(r \geq 2)$ be the given points, and $\phi \in C^{n}([\alpha, \beta])$ such that $(\cdot-\alpha)(\beta-\cdot)\left[\phi^{(n+1)}\right]^{2} \in L[\alpha, \beta]$ and $\boldsymbol{w}=\left(w_{1}, \ldots, w_{m}\right), \boldsymbol{x}=\left(x_{1}, \ldots, x_{m}\right)$ and $\boldsymbol{y}=\left(y_{1}, \ldots, y_{m}\right)$ be m-tuples such that $x_{l}, y_{l}$ $\in[\alpha, \beta], w_{l} \in \mathbb{R}(l=1, \ldots, m)$. Also let $H_{i j}$ be the fundamental polynomials of the Hermite basis and the functions $G$ and $\mathfrak{L}$ be defined by (9) and (44) respectively. Then

$$
\begin{array}{r}
\sum_{l=1}^{m} w_{l} \phi\left(x_{l}\right)-\sum_{l=1}^{m} w_{l} \phi\left(y_{l}\right)=\frac{\phi(\beta)-\phi(\alpha)}{\beta-\alpha} \sum_{l=1}^{m} w_{l}\left(x_{l}-y_{l}\right) \\
+\int_{\alpha}^{\beta}\left[\sum_{l=1}^{m} w_{l}\left(G\left(x_{l}, t\right)-G\left(y_{l}, t\right)\right)\right] \sum_{j=1}^{r} \sum_{i=0}^{k_{j}} \phi^{(i+2)}\left(a_{j}\right) H_{i j}(t) d t \\
+\frac{\phi^{(n-1)}(\beta)-\phi^{(n-1)}(\alpha)}{\beta-\alpha} \int_{\alpha}^{\beta} \mathfrak{L}(s) d s+\kappa(\phi ; \alpha, \beta) .
\end{array}
$$


where the remainder $\kappa(\phi ; \alpha, \beta)$ satisfies the estimation

$$
|\kappa(\phi ; \alpha, \beta)| \leq \frac{\sqrt{\beta-\alpha}}{\sqrt{2}}[\Lambda(\mathfrak{L}, \mathfrak{L})]^{\frac{1}{2}}\left|\int_{\alpha}^{\beta}(s-\alpha)(\beta-s)\left[\phi^{(n+1)}(s)\right]^{2} d s\right|^{\frac{1}{2}} .
$$

Proof. The proof is similar to the proof of Theorem 15 in [5].

The integral version of the above theorem can be stated as:

Theorem 3.4. Let $-\infty<\alpha<\beta<\infty$ and $\alpha \leq a_{1}<a_{2} \cdots<a_{r} \leq \beta,(r \geq 2)$ be the given points, and $\phi \in C^{n}([\alpha, \beta])$ such that $(\cdot-\alpha)(\beta-\cdot)\left[\phi^{(n+1)}\right]^{2} \in L[\alpha, \beta]$ and $x, y:[a, b] \rightarrow[\alpha, \beta], w:[a, b] \rightarrow \mathbb{R}$ be continuous functions. Also let $H_{i j}$ be the fundamental polynomials of the Hermite basis and the functions $G$ and $\mathfrak{J}$ be defined by (9) and (45) respectively. Then

$$
\begin{array}{r}
\int_{a}^{b} w(\tau) \phi(x(\tau)) d \tau-\int_{a}^{b} w(\tau) \phi(y(\tau)) d \tau=\frac{\phi(\beta)-\phi(\alpha)}{\beta-\alpha} \int_{a}^{b} w(\tau)(x(\tau)-y(\tau)) d \tau \\
+\int_{\alpha}^{\beta}\left[\int_{a}^{b} w(\tau)(G(x(\tau), t)-G(y(\tau), t)) d \tau\right] \sum_{j=1}^{r} \sum_{i=0}^{k_{j}} \phi^{(i+2)}\left(a_{j}\right) H_{i j}(t) d t \\
+\frac{\phi^{(n-1)}(\beta)-\phi^{(n-1)}(\alpha)}{\beta-\alpha} \int_{\alpha}^{\beta} \mathfrak{J}(s) d s+\tilde{\kappa}(\phi ; \alpha, \beta) .
\end{array}
$$

where the remainder $\tilde{\kappa}(\phi ; \alpha, \beta)$ satisfies the estimation

$$
|\tilde{\kappa}(\phi ; \alpha, \beta)| \leq \frac{\sqrt{\beta-\alpha}}{\sqrt{2}}[\Lambda(\mathfrak{J}, \mathfrak{J})]^{\frac{1}{2}}\left|\int_{\alpha}^{\beta}(s-\alpha)(\beta-s)\left[\phi^{(n+1)}(s)\right]^{2} d s\right|^{\frac{1}{2}} .
$$

Using Theorem 3.2 we obtain the following Grüss type inequalities.

Theorem 3.5. Let $-\infty<\alpha<\beta<\infty$ and $\alpha \leq a_{1}<a_{2} \cdots<a_{r} \leq \beta,(r \geq 2)$ be the given points, and $\phi \in C^{n}([\alpha, \beta])$ such that $\phi^{(n)}$ is monotonic non decreasing on $[\alpha, \beta]$ and let $\mathfrak{L}$ be defined by (44). Then the representation (48) holds and the remainder $\kappa(\phi ; \alpha, \beta)$ satisfies the bound

$$
|\kappa(\phi ; \alpha, \beta)| \leq\left\|\mathfrak{L}^{\prime}\right\|_{\infty}\left\{\frac{\phi^{(n-1)}(\beta)+\phi^{(n-1)}(\alpha)}{2}-\frac{\phi^{(n-2)}(\beta)-\phi^{(n-2)}(\alpha)}{\beta-\alpha}\right\} .
$$

Proof. The proof is similar to the proof of Theorem 17 in [5].

Integral case of the above theorem can be given:

Theorem 3.6. Let $-\infty<\alpha<\beta<\infty$ and $\alpha \leq a_{1}<a_{2} \cdots<a_{r} \leq \beta,(r \geq 2)$ be the given points, and $\phi \in C^{n}([\alpha, \beta])$ such that $\phi^{(n)}$ is monotonic non decreasing on $[\alpha, \beta]$ and let $x, y:[a, b] \rightarrow[\alpha, \beta], w:[a, b] \rightarrow \mathbb{R}$ be continuous functions and 
the functions $G$ and $\mathfrak{J}$ be defined by (9) and (45) respectively. Then we have the representation (50) and the remainder $\tilde{\kappa}(\phi ; \alpha, \beta)$ satisfies the bound

$$
|\tilde{\kappa}(\phi ; \alpha, \beta)| \leq\left\|\tilde{J}^{\prime}\right\|_{\infty}\left\{\frac{\phi^{(n-1)}(\beta)+\phi^{(n-1)}(\alpha)}{2}-\frac{\phi^{(n-2)}(\beta)-\phi^{(n-2)}(\alpha)}{\beta-\alpha}\right\} .
$$

We present the Ostrowski-type inequalities related to generalizations of majorization inequality.

Theorem 3.7. Suppose that all assumptions of Theorem 2.1 hold. Assume $(p, q)$ is a pair of conjugate exponents, that is $1 \leq p, q \leq \infty, 1 / p+1 / q=1$. Let $\left|\phi^{(n)}\right|^{p}$ : $[\alpha, \beta] \rightarrow \mathbb{R}$ be an $R$-integrable function for some $n \in \mathbb{N}$. Then we have:

$$
\begin{gathered}
\mid \sum_{l=1}^{m} w_{l} \phi\left(x_{l}\right)-\sum_{l=1}^{m} w_{l} \phi\left(y_{l}\right)-\frac{\phi(\beta)-\phi(\alpha)}{\beta-\alpha} \sum_{l=1}^{m} w_{l}\left(x_{l}-y_{l}\right) \\
-\int_{\alpha}^{\beta}\left[\sum_{l=1}^{m} w_{l}\left(G\left(x_{l}, t\right)-G\left(y_{l}, t\right)\right)\right] \sum_{j=1}^{r} \sum_{i=0}^{k_{j}} \phi^{(i+2)}\left(a_{j}\right) H_{i j}(t) d t \mid \\
\leq\left\|\phi^{(n)}\right\|_{p}\|\mathfrak{L}\|_{q},
\end{gathered}
$$

where $\mathfrak{L}$ is defined in (44).

The constant on the right-hand side of (54) is sharp for $1<p \leq \infty$ and the best possible for $p=1$.

Proof. The proof is similar to the proof of Theorem 19 in [5].

Integral version of the above theorem can be given as:

Theorem 3.8. Suppose that all assumptions of Theorem 2.2 hold. Assume $(p, q)$ is a pair of conjugate exponents, that is $1 \leq p, q \leq \infty, 1 / p+1 / q=1$. Let $\left|\phi^{(n)}\right|^{p}$ : $[\alpha, \beta] \rightarrow \mathbb{R}$ be an $R$-integrable function for some $n \in \mathbb{N}$. Then we have:

$$
\begin{gathered}
\mid \int_{a}^{b} w(\tau) \phi(x(\tau)) d \tau-\int_{a}^{b} w(\tau) \phi(y(\tau)) d \tau-\frac{\phi(\beta)-\phi(\alpha)}{\beta-\alpha} \int_{a}^{b} w(\tau)(x(\tau)-y(\tau)) d \tau \\
-\int_{\alpha}^{\beta}\left[\int_{a}^{b} w(\tau)(G(x(\tau), t)-G(y(\tau), t)) d \tau\right] \sum_{j=1}^{r} \sum_{i=0}^{k_{j}} \phi^{(i+2)}\left(a_{j}\right) H_{i j}(t) d t \mid \\
\leq\left\|\phi^{(n)}\right\|_{p}\|\mathfrak{J}\|_{q}
\end{gathered}
$$

where $\mathfrak{J}$ is defined in (45).

The constant on the right-hand side of (55) is sharp for $1<p \leq \infty$ and the best possible for $p=1$. 


\section{4. $n$-Exponential Convexity and Exponential Convexity}

We begin this section by giving some definitions and notions which are used frequently in the results. For more details see e.g. [12], [15] and [22].

Definition 4.1. A function $\phi: I \rightarrow \mathbb{R}$ is $n$-exponentially convex in the Jensen sense on $I$ if

$$
\sum_{i, j=1}^{n} \xi_{i} \xi_{j} \phi\left(\frac{x_{i}+x_{j}}{2}\right) \geq 0,
$$

hold for all choices $\xi_{1}, \ldots, \xi_{n} \in \mathbb{R}$ and all choices $x_{1}, \ldots, x_{n} \in$ I. A function $\phi: I \rightarrow \mathbb{R}$ is $n$-exponentially convex if it is $n$-exponentially convex in the Jensen sense and continuous on $I$.

Definition 4.2. A function $\phi: I \rightarrow \mathbb{R}$ is exponentially convex in the Jensen sense on $I$ if it is n-exponentially convex in the Jensen sense for all $n \in \mathbb{N}$.

A function $\phi: I \rightarrow \mathbb{R}$ is exponentially convex if it is exponentially convex in the Jensen sense and continuous.

Proposition 4.3. If $\phi: I \rightarrow \mathbb{R}$ is an n-exponentially convex in the Jensen sense, then the matrix $\left[\phi\left(\frac{x_{i}+x_{j}}{2}\right)\right]_{i, j=1}^{m}$ is a positive semi-definite matrix for all $m \in$ $\mathbb{N}, m \leq n$. Particularly,

$$
\operatorname{det}\left[\phi\left(\frac{x_{i}+x_{j}}{2}\right)\right]_{i, j=1}^{m} \geq 0
$$

for all $m \in \mathbb{N}, m=1,2, \ldots, n$.

Remark 4.1. It is known that $\phi: I \rightarrow \mathbb{R}^{+}$is a log-convex in the Jensen sense if and only if

$$
\alpha^{2} \phi(x)+2 \alpha \beta \phi\left(\frac{x+y}{2}\right)+\beta^{2} \phi(y) \geq 0,
$$

holds for every $\alpha, \beta \in \mathbb{R}$ and $x, y \in I$. It follows that a positive function is logconvex in the Jensen sense if and only if it is 2-exponentially convex in the Jensen sense.

A positive function is log-convex if and only if it is 2-exponentially convex.

Motivated by inequalities (28) and (30), under the assumptions of Theorems 2.3 and 2.4 we define the following linear functionals:

$$
\begin{array}{r}
\digamma_{1}^{H}(\phi)=\sum_{l=1}^{m} w_{l} \phi\left(x_{l}\right)-\sum_{l=1}^{m} w_{l} \phi\left(y_{l}\right)-\frac{\phi(\beta)-\phi(\alpha)}{\beta-\alpha} \sum_{l=1}^{m} w_{l}\left(x_{l}-y_{l}\right) \\
-\int_{\alpha}^{\beta}\left[\sum_{l=1}^{m} w_{l}\left(G\left(x_{l}, t\right)-G\left(y_{l}, t\right)\right)\right] \sum_{j=1}^{r} \sum_{i=0}^{k_{j}} \phi^{(i+2)}\left(a_{j}\right) H_{i j}(t) d t .(56)
\end{array}
$$




$$
\begin{aligned}
& \digamma_{2}^{H}(\phi)= \int_{a}^{b} w(\tau) \phi(x(\tau)) d \tau-\int_{a}^{b} w(\tau) \phi(y(\tau)) d \tau \\
&-\frac{\phi(\beta)-\phi(\alpha)}{\beta-\alpha} \int_{a}^{b} w(\tau)(x(\tau)-y(\tau)) d \tau \\
&-\int_{\alpha}^{\beta}\left[\int_{a}^{b} w(\tau)(G(x(\tau), t)-G(y(\tau), t)) d \tau\right] \\
& \sum_{j=1}^{r} \sum_{i=0}^{k_{j}} \phi^{(i+2)}\left(a_{j}\right) H_{i j}(t) d t .
\end{aligned}
$$

Remark 4.2. Under the assumptions of Theorems 2.3 and 2.4, it holds $\digamma_{i}^{H}(\phi) \geq 0$, $i=1,2$, for all $n$-convex functions $\phi$.

Lagrange and Cauchy type mean value theorems related to defined functionals are given in the following theorems.

Theorem 4.4. Let $\phi:[\alpha, \beta] \rightarrow \mathbb{R}$ be such that $\phi \in C^{n}[\alpha, \beta]$. If the inequalities in (27) $(i=1)$ and (29) $(i=2)$ hold, then there exist $\xi_{i} \in[\alpha, \beta]$ such that

$$
\digamma_{i}^{H}(\phi)=\phi^{(n)}\left(\xi_{i}\right) \digamma_{i}^{H}(\varphi), \quad i=1,2,
$$

where $\varphi(x)=\frac{x^{n}}{n !}$ and $\digamma_{i}^{H}, i=1,2$ are defined by (56) and (57).

Proof. Similar to the proof of Theorem 4.1 in [16].

Theorem 4.5. Let $\phi, \psi:[\alpha, \beta] \rightarrow \mathbb{R}$ be such that $\phi, \psi \in C^{n}[\alpha, \beta]$. If the inequalities in (27) $(i=1)$, (29) $(i=2)$, hold, then there exist $\xi_{i} \in[\alpha, \beta]$ such that

$$
\frac{\digamma_{i}^{H}(\phi)}{\digamma_{i}^{H}(\psi)}=\frac{\phi^{(n)}\left(\xi_{i}\right)}{\psi^{(n)}\left(\xi_{i}\right)}, \quad i=1,2,
$$

provided that the denominators are non-zero and $\digamma_{i}^{H}, i=1,2$, are defined by (56) $\operatorname{and}(57)$.

Proof. Similar to the proof of Theorem 4.2 in [16].

Now we will produce $n$-exponentially and exponentially convex functions applying defined functionals. We use an idea from [22]. In the sequel $J$ will be interval in $\mathbb{R}$.

Theorem 4.6. Let $\Omega=\left\{\phi_{t}: t \in J\right\}$, where $J$ is an interval in $\mathbb{R}$, be a family of functions defined on an interval $[\alpha, \beta]$ such that the function $t \mapsto\left[x_{0}, \ldots, x_{n} ; \phi_{t}\right]$ is $n$-exponentially convex in the Jensen sense on $J$ for every $(n+1)$ mutually different points $x_{0}, \ldots, x_{n} \in[\alpha, \beta]$. Then for the linear functionals $\digamma_{i}^{H}\left(\phi_{t}\right)(i=1,2)$ as defined by (56) and (57), the following statements hold:

(i) The function $t \rightarrow \digamma_{i}^{H}\left(\phi_{t}\right)$ is n-exponentially convex in the Jensen sense on $J$ and the matrix $\left[\digamma_{i}^{H}\left(\phi_{\frac{t_{j}+t_{l}}{2}}\right)\right]_{j, l=1}^{m}$ is a positive semi-definite for all $m \in \mathbb{N}, m \leq n, t_{1}, . ., t_{m} \in J$. Particularly,

$$
\operatorname{det}\left[\digamma_{i}^{H}\left(\phi_{\frac{t_{j}+t_{l}}{2}}\right)\right]_{j, l=1}^{m} \geq 0 \text { for all } m \in \mathbb{N}, m=1,2, \ldots, n \text {. }
$$


(ii) If the function $t \rightarrow \digamma_{i}^{H}\left(\phi_{t}\right)$ is continuous on $J$, then it is n-exponentially convex on $J$.

Proof. The proof is similar to the proof of Theorem 23 in [5].

The following corollary is an immediate consequence of the above theorem.

Corollary 4.7. Let $\Omega=\left\{\phi_{t}: t \in J\right\}$, where $J$ is an interval in $\mathbb{R}$, be a family of functions defined on an interval $[\alpha, \beta]$ such that the function $t \mapsto\left[x_{0}, \ldots, x_{n} ; \phi_{t}\right]$ is exponentially convex in the Jensen sense on $J$ for every $(n+1)$ mutually different points $x_{0}, \ldots, x_{n} \in[\alpha, \beta]$. Then for the linear functionals $\digamma_{i}^{H}\left(\phi_{t}\right)(i=1,2)$ as defined by (56) and (57), the following statements hold:

(i) The function $t \rightarrow \digamma_{i}^{H}\left(\phi_{t}\right)$ is exponentially convex in the Jensen sense on $J$ and the matrix $\left[\digamma_{i}^{H}\left(\phi_{t_{j}+t_{l}}\right)\right]_{j, l=1}^{m}$ is a positive semi-definite for all $m \in$ $\mathbb{N}, m \leq n, t_{1}, . ., t_{m} \in J$. Particularly,

$$
\operatorname{det}\left[\digamma_{i}^{H}\left(\frac{\phi_{\frac{t_{j}+t_{l}}{2}}}{2}\right]_{j, l=1}^{m} \geq 0 \text { for all } m \in \mathbb{N}, m=1,2, \ldots, n .\right.
$$

(ii) If the function $t \rightarrow \digamma_{i}^{H}\left(\phi_{t}\right)$ is continuous on $J$, then it is exponentially convex on $J$.

Corollary 4.8. Let $\Omega=\left\{\phi_{t}: t \in J\right\}$, where $J$ is an interval in $\mathbb{R}$, be a family of functions defined on an interval $[\alpha, \beta]$ such that the function $t \mapsto\left[x_{0}, \ldots, x_{n} ; \phi_{t}\right]$ is 2 -exponentially convex in the Jensen sense on $J$ for every $(n+1)$ mutually different points $x_{0}, \ldots, x_{n} \in[\alpha, \beta]$. Let $\digamma_{i}^{H}, i=1,2$ be linear functionals defined by (56) and (57). Then the following statements hold:

(i) If the function $t \mapsto \digamma_{i}^{H}\left(\phi_{t}\right)$ is continuous on $J$, then it is 2-exponentially convex function on $J$. If $t \mapsto \digamma_{i}^{H}\left(\phi_{t}\right)$ is additionally strictly positive, then it is also log-convex on J. Furthermore, the following inequality holds true:

$$
\left[\digamma_{i}^{H}\left(\phi_{s}\right)\right]^{t-r} \leq\left[\digamma_{i}^{H}\left(\phi_{r}\right)\right]^{t-s}\left[\digamma_{i}^{H}\left(\phi_{t}\right)\right]^{s-r}, \quad i=1,2,
$$

for every choice $r, s, t \in J$, such that $r<s<t$.

(ii) If the function $t \mapsto \digamma_{i}^{H}\left(\phi_{t}\right)$ is strictly positive and differentiable on $J$, then for every $p, q, u, v \in J$, such that $p \leq u$ and $q \leq v$, we have

$$
\mu_{p, q}\left(\digamma_{i}^{H}, \Omega\right) \leq \mu_{u, v}\left(\digamma_{i}^{H}, \Omega\right)
$$

where

$$
\mu_{p, q}\left(\digamma_{i}^{H}, \Omega\right)= \begin{cases}\left(\frac{\digamma_{i}^{H}\left(\phi_{p}\right)}{\digamma_{i}^{H}\left(\phi_{q}\right)}\right)^{\frac{1}{p-q}}, & p \neq q, \\ \exp \left(\frac{\frac{d}{d p} \digamma_{i}^{H}\left(\phi_{p}\right)}{\digamma_{i}^{H}\left(\phi_{p}\right)}\right), & p=q,\end{cases}
$$

for $\phi_{p}, \phi_{q} \in \Omega$.

Proof. The proof is similar to the proof of Corollary 2 in [5]. 
Remark 4.3. Note that the results from Theorem 4.6, Corollary 4.7 and Corollary 4.8 still hold when two of the points $x_{0}, \ldots, x_{l} \in[\alpha, \beta]$ coincide, say $x_{1}=x_{0}$, for $a$ family of differentiable functions $\phi_{t}$ such that the function $t \mapsto\left[x_{0}, \ldots, x_{l} ; \phi_{t}\right]$ is an $n$ exponentially convex in the Jensen sense (exponentially convex in the Jensen sense, log-convex in the Jensen sense), and furthermore, they still hold when all $(l+1)$ points coincide for a family of $l$ differentiable functions with the same property. The proofs are obtained by (22) and suitable characterization of convexity.

\section{EXAMPLES}

In this section, we present some families of functions which fulfil the conditions of Theorem 4.6, Corollary 4.7 and Corollary 4.8. This enables us to construct a large families of functions which are exponentially convex. Explicit form of these functions are obtained after we calculate explicit action of functionals on a given family.

Example 5.1. Let us consider a family of functions

$$
\Omega_{1}=\left\{\phi_{t}: \mathbb{R} \rightarrow \mathbb{R}: t \in \mathbb{R}\right\}
$$

defined by

$$
\phi_{t}(x)= \begin{cases}\frac{e^{t x}}{t^{n}}, & t \neq 0, \\ \frac{x^{n}}{n !}, & t=0 .\end{cases}
$$

Since $\frac{d^{n} \phi_{t}}{d x^{n}}(x)=e^{t x}>0$, the function $\phi_{t}$ is $n$-convex on $\mathbb{R}$ for every $t \in \mathbb{R}$ and $t \mapsto \frac{d^{n} \phi_{t}}{d x^{n}}(x)$ is exponentially convex by definition. Using analogous arguing as in the proof of Theorem 4.6 we also have that $t \mapsto\left[x_{0}, \ldots, x_{n} ; \phi_{t}\right]$ is exponentially convex (and so exponentially convex in the Jensen sense). Now, using Corollary 4.7 we conclude that $t \mapsto \digamma_{i}^{H}\left(\phi_{t}\right), i=1,2$, are exponentially convex in the Jensen sense. It is easy to verify that this mapping is continuous (although the mapping $t \mapsto \phi_{t}$ is not continuous for $t=0$ ), so it is exponentially convex. For this family of functions, $\mu_{p, q}\left(\digamma_{i}^{H}, \Omega_{1}\right), i=1,2$, from $(61)$, becomes

$$
\mu_{p, q}\left(\digamma_{i}^{H}, \Omega_{1}\right)= \begin{cases}\left(\frac{\digamma_{i}^{H}\left(\phi_{p}\right)}{\digamma_{i}^{H}\left(\phi_{q}\right)}\right)^{\frac{1}{p-q}}, & p \neq q, \\ \exp \left(\frac{\digamma_{i}^{H}\left(i d \cdot \phi_{p}\right)}{\digamma_{i}^{H}\left(\phi_{p}\right)}-\frac{n}{p}\right), & p=q \neq 0, \\ \exp \left(\frac{1}{n+1} \frac{\digamma_{i}^{H}\left(i d \cdot \phi_{0}\right)}{\digamma_{i}^{H}\left(\phi_{0}\right)}\right), & p=q=0,\end{cases}
$$

where $i d$ is the identity function. By Corollary 4.8, $\mu_{p, q}\left(\digamma_{i}^{H}, \Omega_{1}\right)$ is a monotonic function in parameters $p$ and $q$.

Since

$$
\left(\frac{\frac{d^{n} \phi_{p}}{d x^{n}}}{\frac{d^{n} \phi_{q}}{d x^{n}}}\right)^{\frac{1}{p-q}}(\log x)=x,
$$

using Theorem 4.5 it follows that:

$$
M_{p, q}\left(\digamma_{i}^{H}, \Omega_{1}\right)=\log \mu_{p, q}\left(\digamma_{i}^{H}, \Omega_{1}\right), \quad i=1,2
$$


satisfies

$$
\alpha \leq M_{p, q}\left(\digamma_{i}^{H}, \Omega_{1}\right) \leq \beta, \quad i=1,2 .
$$

So, $M_{p, q}\left(\digamma_{i}^{H}, \Omega_{1}\right)$ is a monotonic mean.

Example 5.2. Let us consider a family of functions

$$
\Omega_{2}=\left\{g_{t}:(0, \infty) \rightarrow \mathbb{R}: t \in \mathbb{R}\right\}
$$

defined by

$$
g_{t}(x)= \begin{cases}\frac{x^{t}}{t(t-1) \cdots(t-n+1)}, & t \notin\{0,1, \ldots, n-1\}, \\ \frac{x^{j} \log x}{(-1)^{n-1-j} j !(n-1-j) !}, & t=j \in\{0,1, \ldots, n-1\} .\end{cases}
$$

Since $\frac{d^{n} g_{t}}{d x^{n}}(x)=x^{t-n}>0$, the function $g_{t}$ is $n$-convex for $x>0$ and $t \mapsto \frac{d^{n} g_{t}}{d x^{n}}(x)$ is exponentially convex by definition. Arguing as in Example 5.1 we get that the mappings $t \mapsto \digamma_{i}^{H}\left(g_{t}\right), i=1,2$ are exponentially convex. Hence, for this family of functions $\mu_{p, q}\left(\digamma_{i}^{H}, \Omega_{2}\right), i=1,2$, from (61), is equal to

$$
\mu_{p, q}\left(\digamma_{i}^{H}, \Omega_{2}\right)=\left\{\begin{array}{cl}
\left(\frac{\digamma_{i}^{H}\left(g_{p}\right)}{\digamma_{i}^{H}\left(g_{q}\right)}\right)^{\frac{1}{p-q}}, & p \neq q, \\
\exp \left((-1)^{n-1}(n-1) ! \frac{\digamma_{i}^{H}\left(g_{0} g_{p}\right)}{\digamma_{i}^{H}\left(g_{p}\right)}+\sum_{k=0}^{n-1} \frac{1}{k-p}\right), & p=q \notin\{0,1, \ldots, n-1\}, \\
\exp \left((-1)^{n-1}(n-1) ! \frac{\digamma_{i}^{H}\left(g_{0} g_{p}\right)}{2 \digamma_{i}^{H}\left(g_{p}\right)}+\sum_{\substack{k=0 \\
k \neq p}}^{n-1} \frac{1}{k-p}\right), & p=q \in\{0,1, \ldots, n-1\} .
\end{array}\right.
$$

Again, using Theorem 4.5 we conclude that

$$
\alpha \leq\left(\frac{\digamma_{i}^{H}\left(g_{p}\right)}{\digamma_{i}^{H}\left(g_{q}\right)}\right)^{\frac{1}{p-q}} \leq \beta, \quad i=1,2 .
$$

So, $\mu_{p, q}\left(\digamma_{i}^{H}, \Omega_{2}\right), i=1,2$ is a mean and by $(60)$ it is monotonic.

Example 5.3. Let

$$
\Omega_{3}=\left\{\zeta_{t}:(0, \infty) \rightarrow(0, \infty): t \in(0, \infty)\right\}
$$

be a family of functions defined by

$$
\zeta_{t}(x)= \begin{cases}\frac{t^{-x}}{(\ln t)^{n}}, & t \neq 1 \\ \frac{x^{n}}{n !}, & t=1 .\end{cases}
$$

Since $\frac{d^{n} \zeta_{t}}{d x^{n}}(x)=t^{-x}$ is the Laplace transform of a non-negative function (see [25]) it is exponentially convex. Obviously $\zeta_{t}$ are $n$-convex functions for every $t>0$.

For this family of functions, $\mu_{t, q}\left(\digamma_{i}^{H}, \Omega_{3}\right), i=1,2$, in this case $[\alpha, \beta] \subseteq \mathbb{R}^{+}$, from (61) becomes

$$
\mu_{t, q}\left(\digamma_{i}^{H}, \Omega_{3}\right)= \begin{cases}\left(\frac{\digamma_{i}^{H}\left(\zeta_{t}\right)}{\digamma_{i}^{H}\left(\zeta_{q}\right)}\right)^{\frac{1}{t-q}}, & t \neq q ; \\ \exp \left(-\frac{\digamma_{i}^{H}\left(i d . \zeta_{t}\right)}{t \digamma_{i}^{H}\left(\zeta_{t}\right)}-\frac{n}{t \ln t}\right), & t=q \neq 1 \\ \exp \left(-\frac{1}{n+1} \frac{\digamma_{i}^{H}\left(i d . \zeta_{1}\right)}{\digamma_{i}^{H}\left(\zeta_{1}\right)}\right), & t=q=1 .\end{cases}
$$


This is monotonous function in parameters $t$ and $q$ by (60).

Using Theorem 4.5 it follows that

$$
M_{t, q}\left(\digamma_{i}^{H}, \Omega_{3}\right)=-L(t, q) \ln \mu_{t, q}\left(\digamma_{i}^{H}, \Omega_{3}\right), \quad i=1,2 .
$$

satisfy

$$
\alpha \leq M_{t, q}\left(\digamma_{i}^{H}, \Omega_{3}\right) \leq \beta, \quad i=1,2 .
$$

This shows that $M_{t, q}\left(\digamma_{i}^{H}, \Omega_{3}\right)$ is mean for $i=1,2$. Because of the above inequality (60), this mean is also monotonic. $L(t, q)$ is logarithmic mean defined by

$$
L(t, q)= \begin{cases}\frac{t-q}{\log t-\log q}, & t \neq q \\ t, & t=q .\end{cases}
$$

Example 5.4. Let

$$
\Omega_{4}=\left\{\gamma_{t}:(0, \infty) \rightarrow(0, \infty): t \in(0, \infty)\right\}
$$

be a family of functions defined by

$$
\gamma_{t}(x)=\frac{e^{-x \sqrt{t}}}{t^{n}}
$$

Since $\frac{d^{n} \gamma_{t}}{d x^{n}}(x)=e^{-x \sqrt{t}}$ is the Laplace transform of a non-negative function (see [25]) it is exponentially convex. Obviously $\gamma_{t}$ are $n$-convex function for every $t>0$. For this family of functions, $\mu_{t, q}\left(\digamma_{i}^{H}, \Omega_{4}\right), i=1,2$, in this case for $[\alpha, \beta] \in \mathbb{R}^{+}$, from (61) becomes

$$
\mu_{t, q}\left(\digamma_{i}^{H}, \Omega_{4}\right)= \begin{cases}\left(\frac{\digamma_{i}^{H}\left(\gamma_{t}\right)}{\digamma_{i}^{H}\left(\gamma_{q}\right)}\right)^{\frac{1}{t-q}}, & t \neq q \\ \exp \left(-\frac{\digamma_{i}^{H}\left(i d \cdot \gamma_{t}\right)}{2 \sqrt{t} \digamma_{i}^{H}\left(\gamma_{t}\right)}-\frac{n}{t}\right), & t=q .\end{cases}
$$

This is monotonous function in parameters $t$ and $q$ by (60).

Using Theorem 4.5 it follows that

$$
M_{t, q}\left(\digamma_{i}^{H}, \Omega_{4}\right)=-(\sqrt{t}+\sqrt{q}) \ln \mu_{t, q}\left(\digamma_{i}^{H}, \Omega_{4}\right), \quad i=1,2
$$

satisfy

$$
\alpha \leq M_{t, q}\left(\digamma_{i}^{H}, \Omega_{4}\right) \leq \beta, \quad i=1,2 .
$$

This shows that $M_{t, q}\left(\digamma_{i}^{H}, \Omega_{4}\right)$ is mean for $i=1,2$. Because of the above inequality (60), this mean is also monotonic. 


\section{REFERENCES}

[1] Adil Khan, M., Latif, N., Perić, I., and Pečarić, J., "On Sapogov's extension of Čebyšev's inequality", Thai J. Math., 10(2) (2012), 617-633.

[2] Adil Khan, M., Latif, N., Perić, I., and Pečarić, J., "On majorization for matrices", Math. Balkanica, 27(2) (2013), 3-19.

[3] Adil Khan, M., Niezgoda, M., and Pečarić, J., "On a refinement of the majorization type inequality", Demonstratio Math., 44(1) (2011), 49-57.

[4] Adil Khan, M., Khalid, S., and Pečarić, J., "Refinements of some majorization type inequalities", J. Math. Inequal., 7(1) (2013), 73-92.

[5] Adil Khan, M., M., Latif, N., and Pečarić, J., "Generalization of majorization theorem", $J$. Math. Inequal., 9(3) (2015), 847-872.

[6] Adil Khan, M., Latif N., and Pečarić, J., "Generalization of majorization theorem by Hermite's polynomial", J. Adv. Math. Stud., 8(2) (2015), 206-223.

[7] Adil Khan, M., Latif, N., and Pečarić, J., "Generalization of majorization theorem Via AbelGontscharoff Polynomial", Rad HAZU, to appear.

[8] Adil Khan, M., Latif N., and Pečarić, J., "On generalizations of majorization inequality", Nonlinear Functional Analysis and Applications, 20(2) (2015), 301-327.

[9] Agarwal R. P., Wong P. J. Y., Error Inequalities in Polynomial Interpolation and Their Applications, Kluwer Academic Publishers, Dordrecht/ Boston/London, 1993.

[10] Barnett N. S., Cerone P., Dragomir S. S., "Majorisation inequalities for Stieltjes integrals", Appl. Math. Lett., 22 (2009), 416-421.

[11] Beesack P. R. , On the Green's function of an N-point boundary value problem, Kluwer Academic Publishers, Dordrecht/Boston/London, 1993.

[12] Bernstein, S. N. , "Sur les fonctions absolument monotones", Acta Math., 52 (1929), 1-66.

[13] Cerone P., Dragomir, S. S., "Some new Ostrowski-type bounds for the Čebyšev functional and applications", J. Math. Inequal. 8(1) (2014), 159-170.

[14] Fuchs L., "A new proof of an inequality of Hardy-Littlewood-Polya", Mat. Tidsskr, B (1947), 53-54.

[15] Jakšetić J., Pečarić, J., "Exponential convexity method", J. Convex Anal. no. 1 (2013), 181-197.

[16] Jakšetić J., Pečarić J., Perušić A., "Steffensen inequality, higher order convexity and exponential convexity", Rend. Circ. Mat. Palermo 63 (1) (2014), 109-127.

[17] Latif, N., Pečarić, J., and Perić, I., "On Majorization, Favard and Berwald's Inequalities", Annals of Functional Analysis, 2(1) (2011), 31-50.

[18] Maligranda L., Pečarić J., Persson L. E., "Weighted Favard's and Berwald's Inequalities", J. Math. Anal. Appl. 190 (1995), 248-262.

[19] A. W. Marshall, I. Olkin and Barry C. Arnold, Inequalities: Theory of Majorization and Its Applications (Second Edition), Springer Series in Statistics, New York, 2011.

[20] Niezgoda M., "Remarks on convex functions and separable sequences", Discrete Math., 308 (2008), 1765-1773.

[21] Pečarić, J.,F. Proschan and Y. L. Tong, Convex functions, Partial Orderings and Statistical Applications, Academic Press, New York, 1992.

[22] Pečarić J., Perić J., "Improvements of the Giaccardi and the Petrović inequality and related results", An. Univ. Craiova Ser. Mat. Inform., 39(1) (2012), 65-75.

[23] Pečarić J., "On some inequalities for functions with nondecreasing increments", J. Math. Anal. Appl., 98 (1984), 188-197.

[24] Levin A. Yu., "Some problems bearing on the oscillation of solutions of linear differential equations", Soviet Math. Dokl., 4(1963), 121-124.

[25] Widder D. V., The Laplace Transform, Princeton Univ. Press, New Jersey, 1941.

[26] Widder D. V., "Completely convex function and Lidstone series", Trans. Am. Math. Soc., 51 (1942), 387-398. 